\title{
ERRORS OF REFRACTION AND THEIR SEQUELÆ.
}

\author{
By R. C. DAVENPORT, F.R.C.S.,
}

Ophthalmic Surgeon, Princess Elizabeth of York Hospital for Children; Assistant Surgeon, Royal London Ophthalmic Hospital, Moorfields, \&cc.

It is not intended that this article shall in any way resemble a text book account of the errors of refraction covering, as such would, the necessary anatomy, physiology, and optics. More is the idea to review errors as a whole, and then the main practical issues of the different types.

In theory no doubt any existing refractive error should be found and treated, so that the individual may enjoy the most perfect optical conditions possible. But it must always be borne in mind that the eyes are only part of the living organism, dependent on the whole, deriving from it and adding to it. This may modify the position.

A comfortable and efficient ocular mechanism depends on much more than an accurate optical system. The variable factor of physiological fatigue plays a large part as in any other activity of the organism. Ocular symptoms may be the expression of a general state of affairs, and not due to "eyestrain" at all, and as a corollary to this, an uncorrected error of refraction may in some cases play a large part in symptoms not directly connected with the eyes. It is obviously right that an error of refraction should be sought for in any individual whose signs or symptoms could suggest the presence of such, or where treatment has failed to relieve symptoms even though they may seem remote from the eyes. If any error be found, however, its significance and treatment must be carefully judged in each individual case. No one would deny that glasses should be prescribed for the case with a gross error leading to defective vision, obvious symptoms, or both. It is in the case of the patient with a small error where sound judgment is needed, and it is as unbalanced to suggest that such an error never needs correction as to insist on it being the cause of most of the ills that mankind is heir to. Perhaps the latter is the direction more commonly taken, and there must be endless cases where glasses have been ordered for a small error of refraction to the exclusion of treatment for a much greater derangement of the patient's affairs. Apart from consideration of the error of refraction in relation to the patient's state as a whole, other factors, social and economic, have sometimes to be considered quite seriously before the individual should be advised to wear spectacles. Opinion must often be given where the patient almost insists on getting glasses, or having a change made in an existing correction. Wearisome though it may sometimes be, each case must be judged on its own merits for the real good of the individual. As a side issue, these considerations make a very strong argument against the prescribing of glasses by opticians who, however good may be their refraction work, have not the training to assess every case as a whole rather than as a limited optical one.

With these general points in mind the particular errors may be reviewed.

Hypermetropia, or long sight, is the state, usually due to the smallness of the eye, where parallel rays are brought to a focus behind the retina. In myopia, or short sight, the opposite holds, and here most cases are due to an increase in the normal antero-posterior measurement of the eye. Both these optical defects can be corrected by a spherical lens. In astigmatism, however, the defect may be described as the state where rays on different meridia are not brought to focal points on the same plane, and then a cylindrical lens has to be employed, with or without the addition of a spherical lens. Thus the astigmatism may be hypermetropic, myopic, or mixed. 
Except in very rare cases the infant eye is hypermetropic, so that such a state may be considered physiological, and only an error of refraction in so far as the eye fails to develop to the extent of reaching the emmetropic normal. Probably over 50 per cent. of adults are to some extent hypermetropic. It is perhaps only where the individual has signs or symptoms that the state of affairs should be called an "error" of refraction. In hypermetropia, symptoms in the main play a far greater part than the objective sign of defective vision. Accommodation of the eye is very powerful in the child, and wanes through life, but so long as sufficient is retained, good vision for distance, and even near, can be obtained by its use in the hypermetropic eye except in cases of gross degree. This is explained by the fact that accommodation by its refractive effect will bring forward the focal point of the rays entering the eye.

It is the constant employment of accommodation, and especially the excessive amount called for by near work, that produces the symptoms. The patient, child or adult, will state that vision is excellent, but that after reading, etc., for a while, the print runs together or blurs, and that then or afterwards the eyes will ache, and commonly the head ache too. The symptoms may only appear occasionally, especially with depressed general health or an unwonted amount of near work, and correction of the error, in general for near work only, must depend mainly on how much the symptoms are worrying the patient, either obviously by eyeache or headache, or less obviously as an irritant factor in the general well being.

The use of glasses undoubtedly tends to make the eyes dependent on their help, but quite often especially in the young where the degree of hypermetropia frequently lessens, and familiarity with print increases, glasses need only be worn over a limited period for the symptoms to disappear and not return under satisfactory conditions. In the higher degrees of error glasses are more frequently needed for constant wear, and sooner or later are used in this way. This use of glasses is essential in cases of squint, so commonly associated with hypermetropia. This condition is considered at length, however, in another article.

Myopia presents a somewhat different picture. Here the objective evidence of defective vision is the striking feature, for accommodation of the eye cannot re-adjust the focus as in hypermetropia. Symptoms of fatigue and strain are not, however, infrequent in the higher degrees. Myopia must be regarded as a definitely pathological state, for it means the passing from the normal hypermetropia of infancy to a faulty focus, and is largely due to undue stretching of the developing eye. Rarely a young infant is seen with a high degree of myopia, and especially where this is unilateral it may be considered as a congenital anomaly. Much more commonly the child in the earlier school years is seen to read at an unusually close range, or is discovered to have difficulty in reading the blackboard writing, or seeing distant objects. In spite of this difficulty, the child's defect is quite often unnoticed until there is present an appreciable amount of myopia, and parents are frequently surprised at the extent of defect that an examination on distant test types will reveal. Accepting the condition as a pathological entity, the question of ætiology is at once raised. Endless factors have been put forward, and it is impossible to discuss them in full. It must suffice to say that the hereditary factor, capricious though it may be, is an important one, and some defect in the consolidation of the fibrous tissue is probably the determining element. Thus the onset of myopia may coincide with a period of ill-health or a period of very rapid growth in the developing child. Over-use of the eyes for near work is not so much a cause of myopia as a very real cause of progression in degree once myopia has appeared, especially if such close work is over-indulged in when the child has rapid growth, ill-health, or some other strain to contend with. 
Myopia is in the main a progressive condition through part or most of the growing years. This feature has led to its division into two types, school myopia when it tends to be a slow orderly increase in the degree of myopia over some years of growth, and "malignant" myopia where there is a very rapid uncontrolled progress over a short period. This division is probably unsound. Fortunately, the "malignant" type is rare and there is no doubt that cases of potentially rapid increase can often be detected in the early stages, and by judicious handling kept in check. It is most important to effect this for the eye that has become highly myopic is not an eye that "wears well". Patients with low degrees of myopia suffer only by reason of the distant defect in vision, readily corrected by suitable glasses. Not so in the higher degrees, where not only is vision seldom made perfect by glasses, but where even quite early in adult life degenerative changes appear. These are mainly the formation of floating opacities in the vitreous, and areas of fundus atrophy especially in the central parts where critical visual acuity is undertaken. The stretched choroid splits, or atrophies from impaired blood supply, and choroid and retina degenerate with, of course, gross impairment of vision. Detachment of the retina, too, is a most serious complication of myopia, although the degree of myopia together with other ætiological factors make a less definitely constant clinical picture. Its seriousness and its frequent association with myopia make it a menace which must always be in the background of a case of a higher degree of myopia. Perhaps, too, much panic has produced the advice frequently given to high myopes that they must avoid all strain and violent activity. Such advice and severe limitation of close work leaves very little that can be done by the unfortunate patient. Yet so serious are the consequences that some attention must be called to the risks of violence being the precipitating factor in the promotion of a myopic detachment of the retina. High diving and strenuous gymnastics may well be forbidden to the more highly myopic.

The treatment of myopia must be considered from two directions. First, the wearing of suitable glasses, and secondly, the following of a suitable régime, ocular and general. No use of glasses with or without other treatment will "cure", the myopia. All that can be hoped for is the correction of the optical defect and the checking or the limiting as much as possible of the progression in the degree of error. In all but the higher degrees of myopia, the lens prescribed should fully correct the error, and such glasses must be worn at least for close work, even though this can be readily managed without their aid. The use in this way tends to make for a good working range, to avoid the increased pull on the eyeballs from over convergence, to exercise the accommodation and thus makes some effort to prevent progress in the degree of myopia. As there is a real visual demand for the glasses at a distance, it becomes a practical matter that the glasses are worn more or less constantly. This constant wear is probably ideal in all but the lowest degrees in children although less strictly so in adults. In the higher degrees the use of glasses and their strength are often an individual question, and can hardly be considered here. At the same time that glasses are advised, advice must be given as to the use of the eyes, and attention drawn to the maintenance of the best possible general health. Myopia children often tend to become voracious readers, and avoid outdoor games, etc., and this régime must be altered. All close work should be done at a good range, in a good position, in good light, and broken where possible by short periods of rest. Reading in bed or late at night are undesirable. The general health must be looked to-tonics, vitamins, etc., being given if there seems any need. On the supposition that deficient laying down of calcium in the sclerotic plays a part in the over-stretching of the eyes, calcium therapy is often advocated. This is all to the good, but calcium metabolism is 
still so little understood that real proof of the effects and value of the treatment does not yet exist. But such treatment is certainly worth trying.

The treatment of myopia without glasses by the use of exercises, massage, etc., is much in vogue at present. It seems quite futile to hope that such will cure myopia, and so correct the focus as to make glasses unnecessary. There is no evidence that the treatment plays any real part in checking progress of the condition, and probably all that it achieves is to train myopic eyes to get a little more out of their unaided visual acuity than can normally be managed. Where, in spite of glasses and a sound régime, the myopia is shewing signs of any rapid increase, more active steps are required. These in their severity depend on the degree of myopia and its rate of progress. It may be necessary to stop all homework, fine sewing, etc. If this fails, it may be necessary to take the child away from school, examinations, and all near work for a term, and send him away to the country under the influence of iron and calcium medication.

Such drastic steps, of course, must not be lightly undertaken, but even they may be wise in the presence of a rapidly increasing myopia especially in a fast growing child.

The treatment of the serious complications cannot be considered within the scope of this paper.

Astigmatism may be hypermetropic, myopic, or be of the type where the opposite axes are on either side of the normal.

In myopic and the higher degrees of hypermetropic astigmatism, the visual acuity is always lowered, but symptoms are not common. The eyes are franklyo defective, and the error being too large to be covered by straining efforts, such are not made. If there are symptoms of strain, glasses should be constantly worno in these cases. The smaller degrees of astigmatism on the hypermetropic side area probably the most productive of strain and symptoms, and as these are often very? varied and vision is good, the error is apt to be overlooked. Headache, ocular fatigue, chronic conjunctivitis, nervous fatigue, and irritability are the more commonly obvious effects of the error though less obvious evidence may be given. Many other ill effects, ocular such as cataract, and general such as epilepsy, dyspepsia, constipation, etc., have been credited with an origin in small uncorrected errors of astigmatism, but the evidence is not convincing to the majority. Allowing for the more extreme views, however, there can be no doubt that the suitable correction of a small error, perhaps long unsuspected, will often make an individual a much happier and fitter person. How constantly the glasses should be worn must be judged in each case. Some patients are quite happy with the eyes corrected only for near work, others definitely need the glasses constantly before the eyes. These latter are commonly nervous, highly critical and sensitive types.

In the handling of each case, care must be taken not to strain at a gnat while swallowing a camel. Not only is conscientious and accurate examination necessary for the finding of the error, but discretion and judgment are as necessary for sound treatment.

Anisometropia, or the state where the refraction of one eye is markedly different from the other, may present a difficult problem.

Each case must be handled as a separate entity, for no common procedure will give comfort in all individuals, and often a comfortable result is only attained by the trial and error principle in correcting the two eyes. One eye may have to be left uncorrected to attain satisfactory working of the eyes, or one or both eyes only partially corrected.

It has been mentioned that accommodation, which normally brings divergent rays of light from a near object to a focal point on the retina. wanes with the 
passing of time. Thus the power to focus near objects fails so much in the normal eye at about the age of 45 that convex spherical glasses need to be worn for reading, etc. This effect is, of course, noticed earlier in the hypermetropic eye, whereas the myopic can still see small print at some point even though it be close to the face. In practice, therefore, the hypermetropic eye will need near glasses, and perhaps also distances ones, the normal eye near glasses, and the myopic eye either distance ones only to be discarded for reading or replaced by a second pair for near objects.

This physiological state of affairs is called presbyopia, and though quite a normal state, often alarms the patient with good vision who may think he is going blind.

It is important in correcting presbyopia to make the correction meet the working range of the individual, and as a general rule to keep the power of the lens as low as possible so as to give as flexible a range of use as can be obtained. People with several ranges such as reading, painting, music, etc., may need more than one pair of lenses, the power of each suited to the special distance.

The type of glasses to be worn is a very large subject. A spherical convex lens is needed for hypermetropia and presbyopia, while the glass is concave in myopia. Astigmatism calls for a cylindrical lens which is plane in one axis with a power either convex or concave in the axis at right angles, and this can correct an irregular focus.

Where two strengths are needed, one for distance and one for near, bifocal glasses can be worn, the lower segments of which are more convex than the upper. Such glasses are difficult to get used to, and are often a source of worry to a sensitive patient. If, however, their use can be mastered, they are a great boon to the individual. Frames are important in allowing the lenses to exert their effect correctly, and rigid frames are most desirable in astigmatism so that the axis of the cylindrical lens may be kept accurately placed by the rigidity of the frame. As a further safeguard the lenses should not be round, as this gives scope for rotation in the frame. The frames too play a part in the centering of the lenses, that is to say the relation of the centre of the lens to the visual axis of the eye. This is a most important point, and leads on to considering the use of prism lenses. Such are occasionally used in cases of muscle imbalance, i.e., heterophoria. As the eyes normally converge to a near object, although parallel for distance, it is easy to see that there may be a good deal of licence in the normal balance on the vertical plane, and it is only certain cases that need prisms incorporated into their glasses to correct such imbalance.

On a horizontal plane, however, the eyes should always be perfectly aligned, and any imbalance in this direction more usually needs correction.

An uncorrected muscle imbalance may give rise to a great deal of trouble, and should be suspected, and, of course, sought for at any examination of the eyes. Weakness and fatigue of convergence, or one visual axis higher than the other, usually produce a great deal of ocular strain and discomfort, but the experience of most oculists would be that cases with symptoms caused by the need of a prism in the lens are quite infrequent.

Contact glasses must just be mentioned although their sphere of usefulness is very limited. They consist of thin shells of glass fitting on the front of the eyeball, and thus able to re-adjust entirely the curvature of the cornea and the refraction of the eye. In certain cases of gross astigmatism, and in cases where wearing glasses is undesirable, contact glasses may prove of great value, but only certain individuals will tolerate them sufficiently well for their use to be a practicable matter. They are almost invisible when in place, and in spite of appearing dangerous are not really so. 